\title{
Light-Controlled Polarization of MM-Waves with Photo-Excited Gratings in a Resonant Semiconductor Slab
}

\author{
Vladimir Yurchenko ${ }^{1 *}$, Tugba Navruz ${ }^{1}$, Mehmet Ciydem $^{2}$, Ayhan Altintas $^{3}$ \\ ${ }^{1}$ Electrical and Electronics Engineering Department, Gazi University, Ankara, Turkey \\ ${ }^{2}$ Engitek Engineering Technologies Ltd, Ankara, Turkey \\ ${ }^{3}$ Electrical and Electronics Engineering Department, Bilkent University, Ankara, Turkey \\ *corresponding author, E-mail: v.yurchenko.nuimegmail.com
}

\begin{abstract}
We investigated photoconductive gratings in resonant semiconductor layers acting as light-controlled polarizers of millimeter (MM) waves. We compared the effects of striplike, wire-like, and fin-like gratings excited by red light and IR radiation in Silicon wafers, respectively. The fin-like gratings are shown to be the preferred structures that can operate at limited light intensity. The light-sensitive shift of maxima of transmitted power and polarizing efficiency towards the lower frequency band is observed. The effect makes photoconductive gratings and similar patterns potentially suitable for the design of light-controlled frequencytuning and frequency-modulating components of resonant quasi-optical devices.
\end{abstract}

\section{Introduction}

Optical control of $\mathrm{THz}$ and MM-wave quasi-optical beams is a promising technique for ultra-fast modulation, steering, focusing, and other processing of electromagnetic radiation [1-5]. The technique presents numerous opportunities exploiting ultra-fast dynamics, variability, versatility, and the generic character of optical excitation [1]. The most common realization of the technique is the optical excitation of electron-hole plasma in photo-active semiconductors including uniform layers [1], built-in and photo-excited structures [2-4], and metamaterials [5].

One of many benefits of this approach is the excitation of digitally controlled, optically reconfigurable, photoconductive patterns of diffractive elements in semiconductor wafers [1-3]. As an example, patterns of different kinds can easily be created by using commercially-available digital light processing projectors [3]. In this way, ignoring the limitations in the operation speed, a photo-induced polarizer with tunable polarization angle has been demonstrated, showing a $3 \mathrm{~dB}$ extinction ratio for $\mathrm{THz}$ waves at the frequency of $585 \mathrm{GHz}$.

Optical technology is particularly promising for the dynamic control of $\mathrm{THz}$ metamaterials [5]. In addition to electronic control [6], metamaterials allow for the reduction of required optical power while preserving sufficiently high speed of operation. Some representative examples of

The work was presented at the URSI-TR'2018 IX Scientific Congress. this kind include light-sensitive split-ring meta-arrays [7] and optically controlled chiral metamaterials [8]. The latter, according to simulations, exhibit a giant tunable optical activity at various, practically achievable, photo-excitation intensities. This allows for the achievement of active $\mathrm{THz}$ polarization components like tunable polarizers and polarization filters.

Optical control can be used for enhancing functionality of specific devices. As an example, an ultra-fast phase inversion of quasi-optical centimeter-wave beams has been achieved with induced photoconductivity in Silicon [9]. An optically controlled reconfigurable antenna for $5 \mathrm{G}$ broadband cellular communication networks has been produced in [10] by inserting photosensitive semiconductor elements at the critical nodes of the wave radiating structure. By applying the concept of optically induced electromagnetic band gap materials, a reconfigurable $140-220 \mathrm{GHz}$ waveguide filter using a mesa array structure has been proposed and simulated in [11].

Despite the abundance of design possibilities, typical structures require, for their optimal performance, a very high level of excitation that corresponds to light power flux of $P_{L} \gtrsim 1 \mathrm{MW} / \mathrm{cm}^{2}$ [1-5]. This is an extremely high power that can only be applied in a pulse mode at a low pulse repetition frequency, that makes many high-speed applications questionable.

We are interested, however, in the diffraction structures that can be operated at a moderate speed and limited optical power in order to achieve a reasonable trade-off between these two competing parameters. The simplest structures, which are suitable for our analysis, are gratings that can be used as the polarizers of electromagnetic waves. It is the gratings of this kind, which are created by the illumination of intermediate intensity, that are going to be investigated in this research.

A representative case of photo-excited gratings created on a GaAs surface for manipulation of $\mathrm{THz}$ waves has been investigated recently in [12]. The high-contrast pattern of photo-excited carriers presents a dynamical $\mathrm{THz}$ component (a polarizer) with reconfigurable functionality. When using more sophisticated patterns, one can produce, e.g., diffractive meta-surfaces with focusing capabilities [13] and other devices, which acquire their functionality under illumination. 
Diffractive gratings in [12] also required high-power lasers for their excitation. Specifically, they were excited by femtosecond pulses having energy fluence in the range of $\Phi=32-156 \mu \mathrm{J} / \mathrm{cm}^{2}$. Should the light pulses of duration $t_{P}=1 \mathrm{fs}$ and characteristic fluence of $\Phi=100 \mu \mathrm{J} / \mathrm{cm}^{2}$ have been used at the repetition frequency $f_{R E P}=10 \mathrm{GHz}$ (the duty cycle of $0.001 \%$ ), the total light power flux would be as high as $P_{L}=1 \mathrm{MW} / \mathrm{cm}^{2}$. This is, clearly, much too high power flux for conventional applications, e.g., in telecommunications.

For comparison, the light of commercially available $100 \mathrm{~W}$ LED arrays at $100 \%$ efficiency, when focused on an area of only $A=1 \mathrm{~cm}^{2}$, would create a power flux of $P_{L}=0.1 \mathrm{~kW} / \mathrm{cm}^{2}$, that looks more acceptable. Yet, this value is, approximately, a thousand times greater than the maximum Sun light power flux at the Earth surface.

The sensitivity of devices can be increased by the use of quasi-optical resonators $[14,15]$. The structures in $[14,15]$ operate as beam switches for $\mathrm{THz}$ and $\mathrm{MM}$ waves at certain resonant frequencies. When using diffractive photo-excited patterns in a semiconductor layer, one would expand the functionality of these devices. The illumination necessary for the operation of the devices can be created by LED arrays rather than the high-power lasers.

An issue, though, arises about the resonant operation of these devices at various light intensities. The matter is that, unlike the GaAs layer in [12], a semiconductor in this setting should behave as a resonator at any accepted level of illumination.

The basic model for the analysis of these effects is a semiconductor layer of resonant (half-wavelength) thickness with photo-excited (and partially diffused) gratings created either near the surface or deeper inside the wafer. The gratings can be considered as quasi-optical polarizers for MM waves.

The aim of this work is to investigate the effects of photoconductive gratings in a resonant semiconductor layer created by light of moderate intensity and operated as lightcontrolled polarizers for the MM-wave beams.

In general, laser-induced gratings and related phenomena have been investigated in various materials for many decades after the invention of lasers [16-18]. Typical issues of interest were various nonlinear effects, the high-speed dynamics, and the measurements of relaxation parameters of materials, mostly, semiconductors, which are observed under the strong illumination specific for those experiments [19-23].

Unlike the former research, the primary goal of this work is the analysis of the interplay between the effects of transmission, reflection, scattering, and absorption of electromagnetic waves under the mildly resonant conditions in a semiconductor wafer with moderate periodic spatial variation of photoconductivity and, therefore, complex dielectric constant, respectively, which is supposed to be just sufficient for the emerging functionality of structures as the light-controlled polarizers of MM-wave beams.

\section{Photo-Excited Gratings of Photoconductivity in a Semiconductor Slab}

We consider the problem of MM wave propagation through a Silicon $(\mathrm{Si})$ wafer of thickness $d(0<x<d)$ covered on the front side $(x=0)$ with a grating mask of black dielectric film having narrow air slots for the excitation of a periodic photoconductive grating inside the wafer (Fig. 1). The mask is assumed to be opaque for both visible and infrared (IR) radiation but transparent for MM waves. Photoexcitation is produced by the LED array light source creating a uniform illumination of the front surface of the given structure. The propagation of MM waves through such a structure is then investigated (Figs. $2-4$ ) and polarization efficiency is evaluated (Figs. $5-6$ ).

We compare the effects arising in (i) strip-like, (ii) wirelike, and (iii) fin-like photoconductive gratings as shown in Fig. 1. The gratings are produced by photo-excitation of the Si wafer with (i, ii) red light (630 nm wavelength) and (iii) infrared (IR) radiation (1000 $\mathrm{nm}$ wavelength) assuming the wafer thickness $d=0.5 \mathrm{~mm}$. A Si wafer at this thickness is resonant-transparent for MM waves at the frequency $f=$ $90 \mathrm{GHz}$, which is one of the frequencies of interest in the $\mathrm{W}$ frequency band.

The other parameters are the electron-hole recombination length $L=0.02 \mathrm{~mm}$ (the electron-hole lifetime $\tau=0.22 \mu \mathrm{s}$ ), the grating period $p=0.3 \mathrm{~mm}$, and the air slots in the grating mask (i) $c=0.1 \mathrm{~mm}$ and (ii, iii) $c=0.02 \mathrm{~mm}$. Rapid surface recombination is assumed at $x=0$ and $x=d$, which is typical for Si wafers.

Once considering polarizer structures, we choose the grating period to be smaller than the MM wave radiation wavelength. The parameters above satisfy this condition in the frequency band of $f=10-150 \mathrm{GHz}$ considered below. The recombination length $L$ is chosen to be small as compared to the grating period $p$ so that electron-hole diffusion could not smear out the photoconductivity profile. At the same time, the recombination length is not too small so that significant photoconductivity contrast and, therefore, sufficient magnitude of the effects could be observed at moderate light intensity.

Light absorption in a $\mathrm{Si}$ wafer is specified by the frequency-dependent absorption length $l(\nu)$, the quantum flux spectral density $q(\nu)$ of the light source, and the light power reflection coefficient at the semiconductor surface $r(\nu)$ where $\nu$ is the light frequency. The quantum yield of the light absorption is assumed to be $\eta=1$. The light absorption length $l(\nu)$ is a well-known tabulated quantity [24]. The quantum flux spectral density $q(\nu)$ is found from the power spectra of light emitting diodes (LEDs), which are considered as the typical light sources in this problem. Finally, the reflection coefficient $r(\nu)$ is found using the refractive index of Silicon, which is also a well tabulated quantity [24].

Simulations of gratings and wave propagation are made using the FlexPDE software version 7.09. Simulations are organized in two stages.

At the first stage, photoconductivity profiles of the 


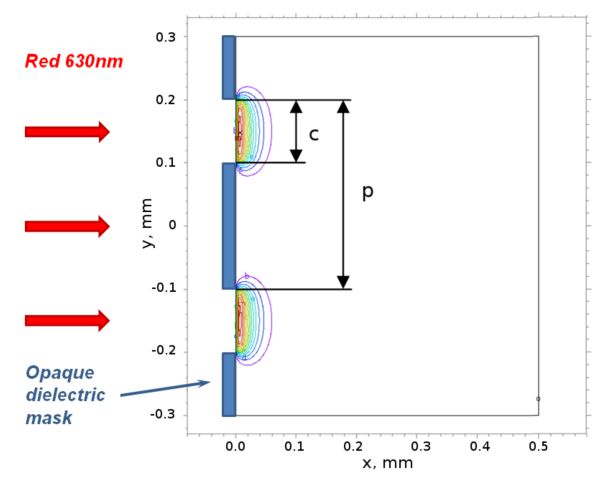

(a)

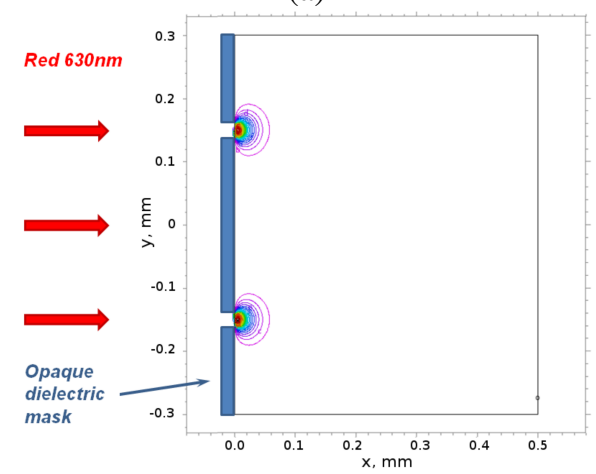

(b)

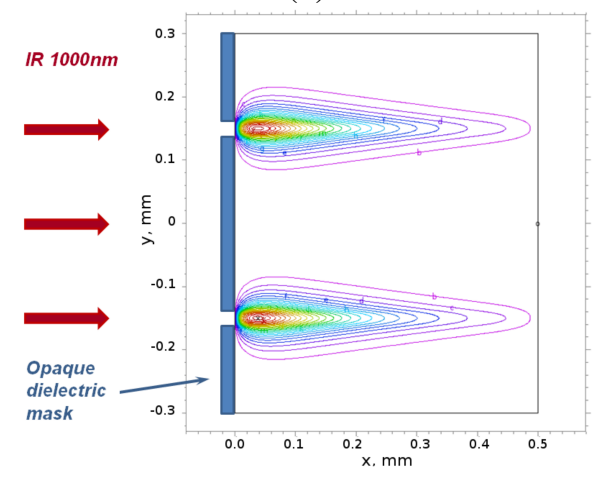

(c)

Figure 1: Photoconductive gratings of (a) strip-like, (b) wire-like, and (c) fin-like profile in a $0.5 \mathrm{~mm} \mathrm{Si}$ wafer excited by $(\mathrm{a}, \mathrm{b})$ red light $(630 \mathrm{~nm})$ and (c) IR radiation $(1000 \mathrm{~nm})$ at the recombination length $L=0.02 \mathrm{~mm}$, the grating period $p=0.3 \mathrm{~mm}$, and the air slots in the grating mask (a) $c=0.1 \mathrm{~mm}$ and (b, c) $c=0.02 \mathrm{~mm}$ (curves show the conductivity distribution).

photo-excited gratings have been computed at the given kind of illumination and the fixed value of the total light power flux $P_{L}$. The latter is chosen to be the same for all cases so as to be used as a reference value in further simulations.

At the second stage, MM wave propagation through the semiconductor slab with photo-excited gratings has been computed. Various cases of light intensity, kinds of gratings, and the incident MM wave polarization have been considered. Normal incidence of the linearly polarized uniform plane MM wave on the front semiconductor surface $(x=0)$ is specified. The cases of $E$ and $H$ polarization have been considered when either the electric $\vec{E}$ field or the magnetic $\vec{H}$ field of the incident wave is parallel to the grating strips, respectively.

The grating strips are aligned along the $z$ axis in the Cartesian frame as used in the grating profile simulations, see Fig. 1. So, the $E$ polarization is the case when the incident wave is defined by the $E_{z}$ component, which is the only non-zero $\vec{E}$ field component of the incident wave. Similarly, the $H$ polarization is specified by the only $H_{z}$ component of the $\vec{H}$ field of the incident wave.

The quantities of interest in the MM wave propagation are the power transmission, reflection, and absorption coefficients $\left(P_{t}=\left|S_{21}\right|^{2}, P_{r}=\left|S_{11}\right|^{2}\right.$, and $P_{a}=1-P_{t}-P_{r}$, respectively, where $S_{21}$ and $S_{11}$ are the relevant scattering matrix coefficients) and the polarization ratio of transmitted and reflected waves, all computed as the functions of the MM wave frequency.

\section{Simulation of Photoconductivity Profiles of Photo-Excited Gratings}

The reference grating profiles were computed at the light power flux $P_{L}=0.1 \mathrm{~kW} / \mathrm{cm}^{2}$. The maxima of electron density $n$, photoconductivity $\sigma$, dielectric loss tangent $\tan (\delta)$, and the minima of skin depth $l_{s}$ for MM waves at $100 \mathrm{GHz}$ as found for gratings (i), (ii), and (iii) at this illumination, are $n=(4 ; 3 ; 2.45) 10^{16} 1 / \mathrm{cm}^{3}$, $\sigma=(1.24 ; 0.95 ; 0.76) \mathrm{kS} / \mathrm{m}, \tan (\delta)=(19 ; 14 ; 12)$, and $l_{s}=(45 ; 51 ; 57) \mu \mathrm{m}$, respectively (the dark-state parameters are $\sigma=3 \mathrm{mS} / \mathrm{m}$ and $\left.\tan (\delta)=10^{-4}\right)$.

We use the electron-hole diffusion equation [25] presented in the form

$$
\nabla(\nabla(n))-\left(n-n_{0}\right) / L^{2}+G(x, y)=0
$$

where $n=n_{0}+\delta n$ is the electron density in a semiconductor under illumination, $n_{0}$ is the dark-state electron density, $\delta n$ is the excess density of non-equilibrium electrons created by photo-excitation, $L=\sqrt{D \tau}$ is the ambipolar diffusion length, $D=2 D_{n} D_{p} /\left(D_{n}+D_{p}\right), D_{n}$ and $D_{p}$ are the electron and hole diffusion coefficients, $\tau$ is the electronhole recombination lifetime, and $G(x, y)$ is the term describing the generation of the electron-hole pairs by the light flux.

Using the quantities defined above, the term $G(x, y)$ is computed as follows

$$
G(x, y)=g(x) g(y)
$$

where $g(y)$ describes the light intensity profile as defined by the shadowing mask with grating slots on the semiconductor surface presented in Fig. 1 and $g(x)$ defines the electronhole generation rate as a function of $x$ when created by the incident light of the given spectral density and the total power flux $P_{L}$,

$$
g(x)=\int l^{-1}(\nu)[1-r(\nu)] q(\nu) \exp [-x / l(\nu)] d \nu .
$$


The integral is taken over the range of frequencies specific for a given light source, of which we consider the red and the IR LEDs with basic wavelength $630 \mathrm{~nm}$ and $1000 \mathrm{~nm}$, respectively.

The boundary conditions require $n=n_{0}$ at both the front and the rare semiconductor surfaces that corresponds to extremely fast surface recombination as mentioned above. The other boundary conditions are imposed at the side borders of the computation domain where zero electron currents are required due to the symmetry of the domain definition.

Equation (1) describes the ambipolar diffusion of nonequilibrium electrons and holes (photo-excited charge carriers) at the density $n$ and $p$, respectively, in an undoped high-resistivity semiconductor under the moderate illumination when $n, p \gg n_{i}$ ( $n_{i}$ is the intrinsic carrier density), $n=p$ (electron-hole plasma is quasi-neutral), and, at the same time, $n \ll N_{c}$ and $p \ll N_{v}$ where $N_{c}$ and $N_{v}$ are the effective densities of states in the conductive and valence bands, respectively (plasma is classical, non-degenerate).

High-power lasers can produce high-density degenerate electron-hole plasma even in a semiconductor with very short electron-hole recombination lifetime $\tau$ and, therefore, small diffusion length $L$ [16-18]. This case, however, is beyond the scope of this research, since we are interested, specifically, in the effects which are accessible at sufficiently low light intensity.

\section{Simulation of MM Wave Scattering by Photo-Excited Gratings}

Using the grating profiles computed above and applying the light power scaling factors, we simulated different cases of MM wave coupling with photo-excited gratings at different levels of light intensity, Figs. 2 - 4. A linear growth of photoconductivity with increasing light intensity occurs at light power fluxes up to $10 \mathrm{~kW} / \mathrm{cm}^{2}$. Up to this power, the electron-hole plasma at the given recombination rate remains non-degenerate, though the growth continues at greater intensities also.

FlexPDE software was used for solving the MM wave propagation and scattering problems. The software is designed for solving partial differential equations (PDE) by the finite element method (FEM) [26]. When applied to electrodynamics, the method requires the wave equation to be presented in a special form.

In this approach, for the automatic compliance with the boundary conditions for tangential components of both the electric and magnetic fields at the semiconductor surfaces, the wave equation should be written in the form

$$
\nabla\left(\mu^{-1} \nabla\left(E_{z}\right)\right)+k_{0}^{2} \varepsilon E_{z}=0
$$

for the $E_{z}$ component in the $E$ polarization problem and

$$
\nabla\left(\varepsilon^{-1} \nabla\left(H_{z}\right)\right)+k_{0}^{2} \mu H_{z}=0
$$

for the $H_{z}$ component in the $H$ polarization case. Here, $k_{0}=\omega / c$ is the free-space wavenumber, $\omega=2 \pi f, c$ is the free-space speed of light, $\mu=1$ is the relative permeability of Silicon, and $\varepsilon=\varepsilon(x, y)$ is the relative permittivity under illumination. The latter is defined as $\varepsilon=\varepsilon_{r}+i \varepsilon_{i}$ where $\varepsilon_{r}$ is the real part of $\varepsilon$, which is the same as in the dark state, $\varepsilon_{i}=\sigma(x, y) / \omega \varepsilon_{0}, \varepsilon_{0}$ is the absolute free-space permittivity, and $\sigma(x, y)$ is the conductivity that increases and acquires the grating profile under illumination.

The incident MM wave is the uniform plane wave of linear polarization propagating in the positive direction along the $x$ axis. The incident wave is introduced via the boundary condition, which is imposed on either the $E_{z}$ or the $H_{z}$ field component, depending on the polarization, at the front surface $x=-X_{L}<0$ of the computation domain. The boundary condition requires the incident wave to enter the computation domain through this surface and propagate towards the semiconductor. At the same time, any waves reflected from the semiconductor should leave the domain with no obstruction.

In a similar way, the radiation condition is formulated at the rare surface of the computation domain $x=X_{R}>d$. The condition requires that no incoming waves could enter the computation domain through this surface. We choose $X_{L}$ and $X_{R}$ equal to about one wavelength of the MM wave that appears sufficient for the accurate solution of these problems.

The other boundary conditions are imposed on the wave fields at the side borders of the computation domain. The side borders are defined at $y= \pm Y_{s}$ in a symmetric manner to include an integer number of grating periods (e.g., two periods in Fig. 1 where $Y_{s}=0.3 \mathrm{~mm}$ ). Then, the conditions require that no waves propagate across the side borders and the field components $E_{z}$ and $H_{z}$, respectively, achieve their extrema.

The simulation results obtained for the transmission, reflection, and absorption spectra of the MM waves of both the $E$ and $H$ polarizations incident on the Si wafers with photo-excited gratings are shown in Figs. $2-4$.

\section{MM wave Polarization with Photo-Excited Gratings}

MM wave propagation is computed for wafers with gratings in the frequency range of $f=10-150 \mathrm{GHz}$. The gratings are of sub-wavelength dimensions at these frequencies. They are of interest for the analysis of light-controlled polarization devices for MM waves. Figs. 5 and 6 present the polarizing efficiency of the gratings specified above.

The polarizing efficiency can be evaluated as the polarization ratio computed with relevant components of transmitted and reflected waves. For gratings with conductive strips parallel to the $z$ axis, it is the quantities $H_{z} / E_{z}$ for transmitted waves and $E_{z} / H_{z}$ for reflected waves that represent the polarizing efficiency, being large for good polarizers.

Simulations show that photo-excited gratings, indeed, can operate as the light-controlled polarizers, whose polarizing efficiency grows with increasing light intensity. Of the 


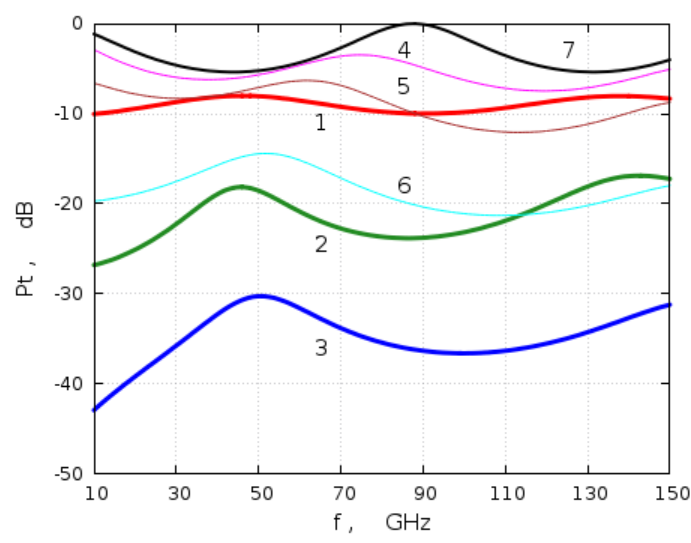

(a)

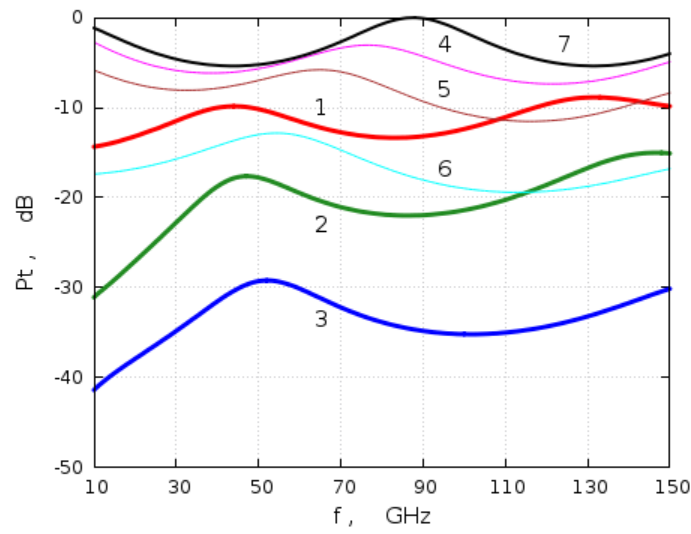

(b)

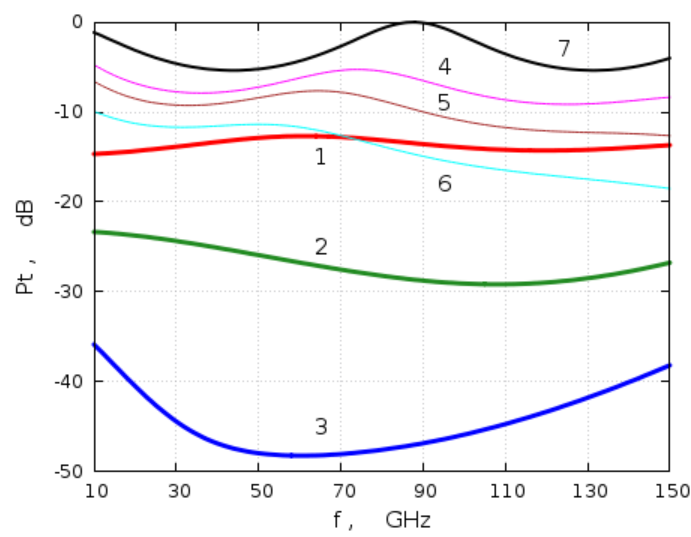

(c)

Figure 2: Transmission through (a) strip-like, (b) wire-like, and (c) fin-like photo-excited gratings for MM waves of $E$ (curves 1 to 3 ) and $H$ (curves 4 to 6) polarization at (a) $P_{L}=0.1,1,10 \mathrm{~kW} / \mathrm{cm}^{2}$, (b) $P_{L}=1,10,100 \mathrm{~kW} / \mathrm{cm}^{2}$, and (c) $P_{L}=0.1,0.3,1 \mathrm{~kW} / \mathrm{cm}^{2}$, respectively (curve 7 shows the dark-state results).

three kinds of gratings, fin-like gratings show much better performance than the other two and the wire-like gratings show the worst performance of all at the same light intensity.

When choosing for comparison the light power flux $P_{L}=1 \mathrm{~kW} / \mathrm{cm}^{2}$, we observe the ratio $H_{z} / E_{z}$ for trans-

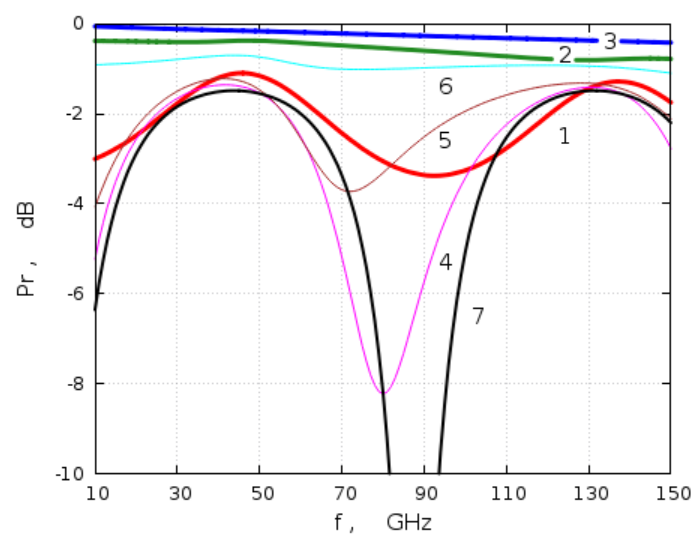

(a)

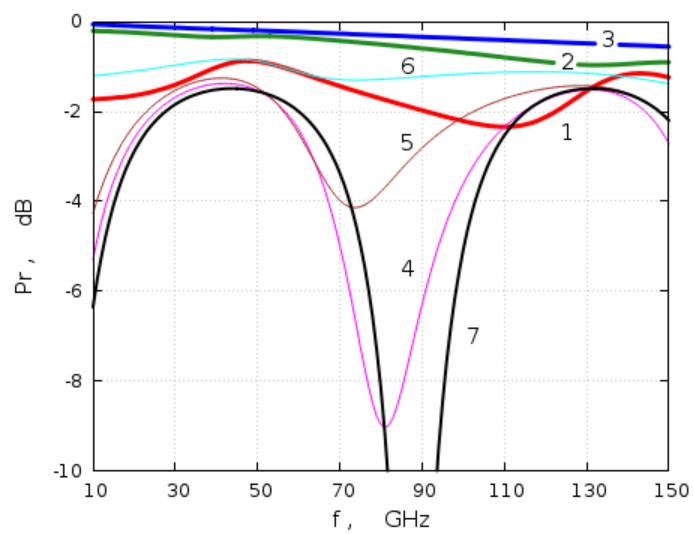

(b)

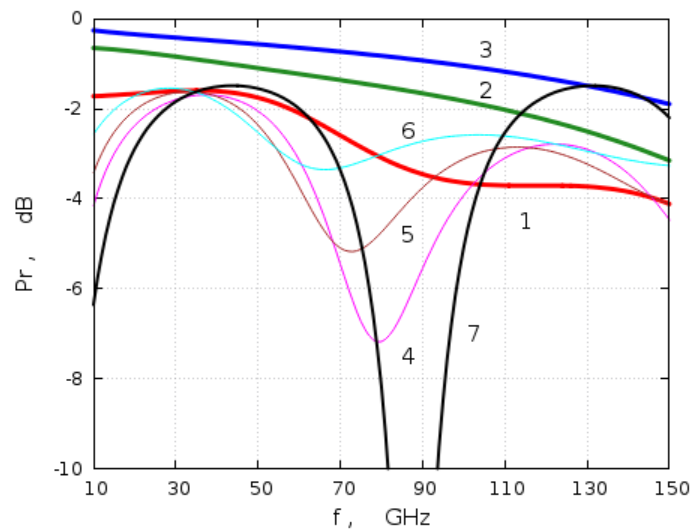

(c)

Figure 3: Reflection from (a) strip-like, (b) wire-like, and (c) fin-like photo-excited gratings for $\mathrm{MM}$ waves of $E$ (curves 1 to 3 ) and $H$ (curves 4 to 6) polarization at (a) $P_{L}=0.1,1,10 \mathrm{~kW} / \mathrm{cm}^{2}$, (b) $P_{L}=1,10,100 \mathrm{~kW} / \mathrm{cm}^{2}$, and (c) $P_{L}=0.1,0.3,1 \mathrm{~kW} / \mathrm{cm}^{2}$, respectively (curve 7 shows the dark-state results).

mitted waves at the representative frequencies of about $f=70 \mathrm{GHz}$ to be, approximately, $15 \mathrm{~dB}, 10 \mathrm{~dB}$, and $35 \mathrm{~dB}$ for the strip-like, wire-like, and fin-like gratings, respectively. This hierarchy of performance should be expected from the general view of grating photoconductivity patterns presented in Fig. 1. Performance in reflection is, 


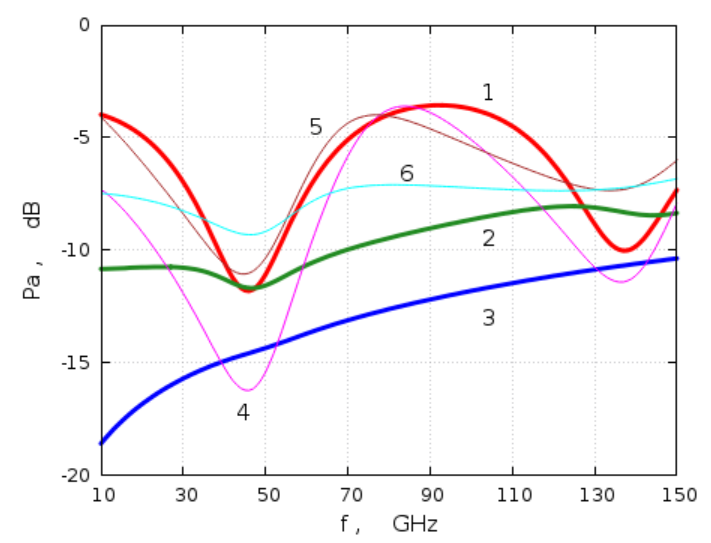

(a)

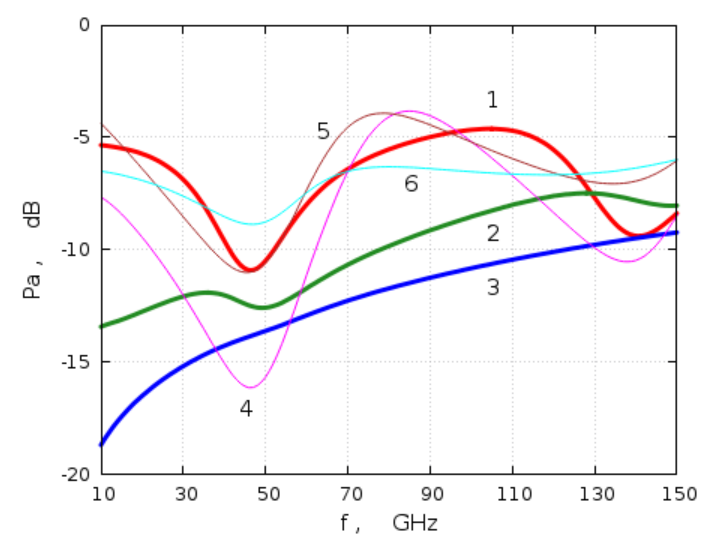

(b)

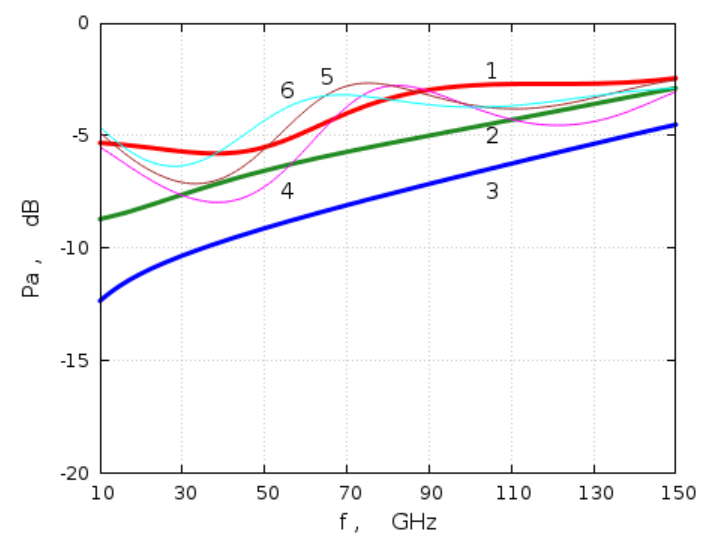

(c)

Figure 4: Absorption in a slab with (a) strip-like, (b) wirelike, and (c) fin-like photo-excited gratings for MM waves of $E$ (curves 1 to 3 ) and $H$ (curves 4 to 6 ) polarization at (a) $P_{L}=0.1,1,10 \mathrm{~kW} / \mathrm{cm}^{2}$, (b) $P_{L}=1,10,100 \mathrm{~kW} / \mathrm{cm}^{2}$, and (c) $P_{L}=0.1,0.3,1 \mathrm{~kW} / \mathrm{cm}^{2}$, respectively (curve 7 shows the dark-state results).

however, low, which is explained by significant reflection of MM waves of all polarizations straight from the semiconductor surface.

A specific feature of grating polarizers in a resonant slab is that all of them and, most of all, the strip-like and the wire-like gratings, Fig. 5 (a) and (b), show a noticeable drop of polarizing efficiency in transmission at certain resonant frequencies. The effect is explained by the resonant transmission of MM waves through a Si wafer. In Figs. 2 and 5 , the resonant transmission frequencies under illumination are $f_{1} \approx 40-50 \mathrm{GHz}$ and $f_{2} \approx 130 \mathrm{GHz}$. For comparison, in a $\mathrm{Si}$ wafer with no grating, the resonant frequencies are $f_{0} \approx 90 \mathrm{GHz}$ in the dark and, similarly, $f_{1} \approx 45 \mathrm{GHz}$ and $f_{2} \approx 130 \mathrm{GHz}$ under illumination (Fig. 7). A similar effect occurs in the $0.25 \mathrm{~mm}$ wafer at twice the frequencies.

The effect is explained by the change of refractive index in photoconductive domains under illumination and related re-distribution of the MM wave field inside the wafer. For an explanation, we should compare the skin-depth in photoconductive domains $\left(l_{s}>50 \mu \mathrm{m}\right.$ at $\left.P_{L}=0.1 \mathrm{~kW} / \mathrm{cm}^{2}\right)$ and the size of the domains defined, essentially, by the electron-hole diffusion length and the grating dimensions ( $L \sim c \sim 20 \mu \mathrm{m}$, while the light absorption length is only $l \sim 3 \mu \mathrm{m}$ for the red light).

We can see that MM waves can easily penetrate into the photoconductive domains under the given illumination. At the same time, photoconductivity $\sigma$ and, therefore, $\varepsilon_{i}$ in the domains is already quite large $\left(\varepsilon_{i} / \varepsilon_{r}>20\right.$ at $f=100 \mathrm{GHz}$ and $P_{L}>0.1 \mathrm{~kW} / \mathrm{cm}^{2}$ ) so that the refractive index $\tilde{n}$ for MM waves increases $\left(\tilde{n}=\operatorname{Re}(\sqrt{\varepsilon}) \approx \sqrt{\varepsilon_{i}} / 2>7\right)$ as compared to the dark-state value $\left(\tilde{n}_{0} \approx 3\right)$.

As a result of these relationships, the effective wafer thickness in terms of the number of wavelengths of MM waves increases with illumination, that makes the halfwavelength transparency to appear at the lower frequencies. The effect is clearly seen in Figs. 2 and 3, (a) and (b), for the $H$ polarized waves (curves 4 to 6 as compared to the dark-state curve 7). This is the case of accepted polarization when the gratings are more transparent.

At higher light intensity, photoconductive domains would become less accessible for MM waves, thus, making the other domains, where the MM wave field is confined, to be smaller. Then, the resonant transparency bands would start shifting towards the higher frequencies. This effect is already visible for the waves of $E$ polarization, see Figs. 2 and 3, (a) and (b), curves 1 to 3. This is the case of rejected polarization when the gratings are less transparent.

The effect of half-wavelength transparency leads to the decrease of polarizing efficiency of photo-excited gratings at the resonant frequencies. This happens because, due to the resonant constructive interference, the structure becomes transparent even for the waves of rejected polarization. At the same time, away from the half-wavelength resonant frequency, photo-excited gratings show quite good polarizing efficiency even at the moderate level of light intensity. The best performance is achieved with fin-like gratings which, due to the significant length of light absorption for $1000 \mathrm{~nm}$ IR radiation, $l=156 \mu \mathrm{m}$, extend across the entire $\mathrm{Si}$ wafer and provide strong coupling with MM waves of the relevant polarization.

The resonant half-wavelength decrease of polarizing efficiency in transmission is the same effect as observed in dual-layer wire-grid polarizers [27]. Away from these fre- 


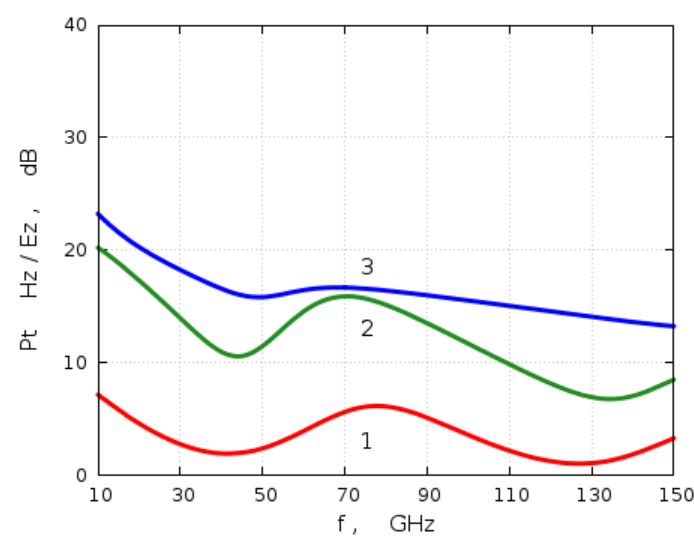

(a)

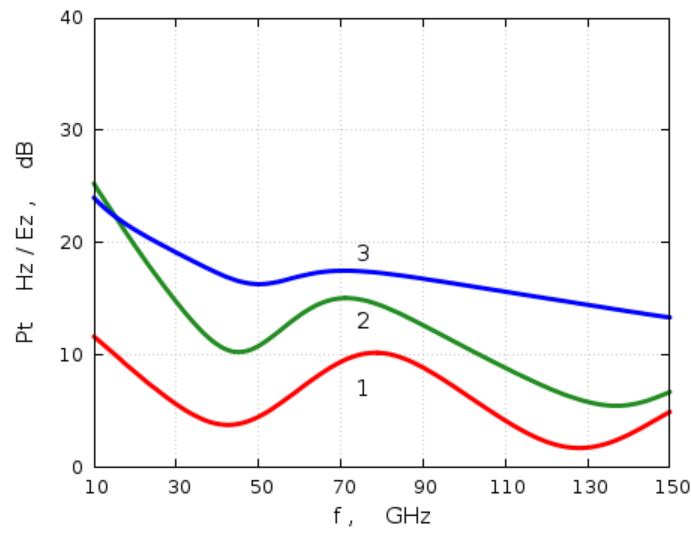

(b)

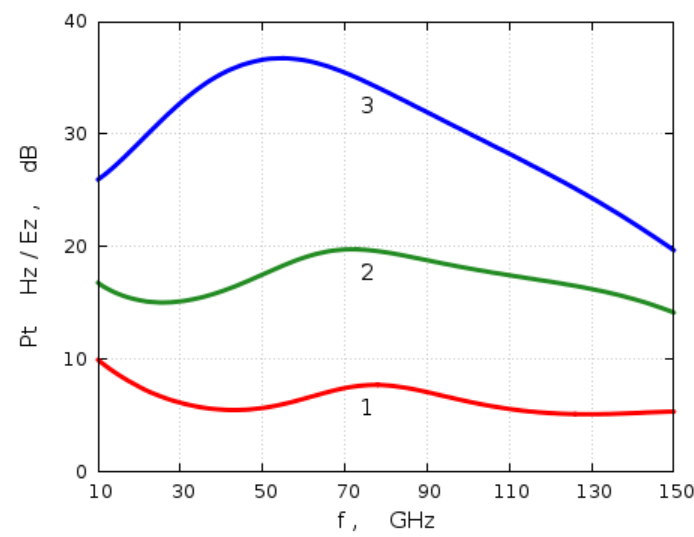

(c)

Figure 5: Polarization ratio for transmitted waves through (a) strip-like $\left(P_{L}=0.1,1,10 \mathrm{~kW} / \mathrm{cm}^{2}\right)$, (b) wire-like $\left(P_{L}=1,10,100 \mathrm{~kW} / \mathrm{cm}^{2}\right)$, and (c) fin-like $\left(P_{L}=\right.$ $0.1,0.3,1 \mathrm{~kW} / \mathrm{cm}^{2}$ ) photo-excited gratings (curves 1 to 3 , respectively).

quencies, however, the polarizing efficiency of dual grids is squared (doubled in decibels) as compared to the case of a single grid. We suppose that, in our fin-like gratings, an enhanced efficiency in transmission observed away from the resonant frequencies is also, partially, related to this effect.

The use of sufficient light intensity is also an important condition. When using extremely high intensity as in [12]

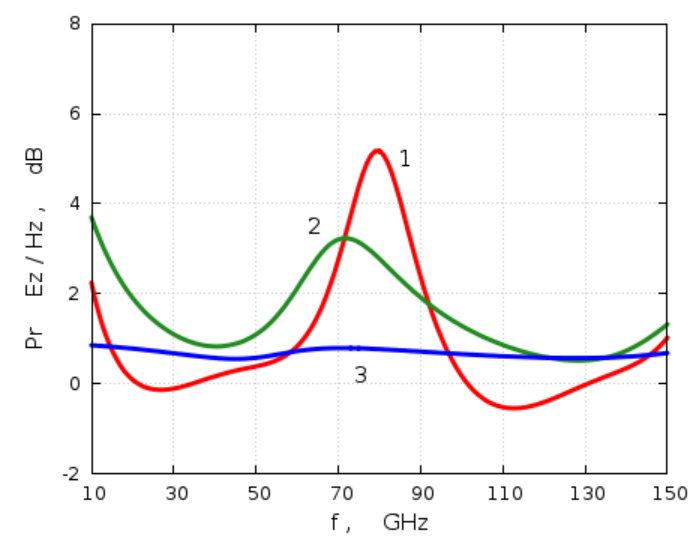

(a)

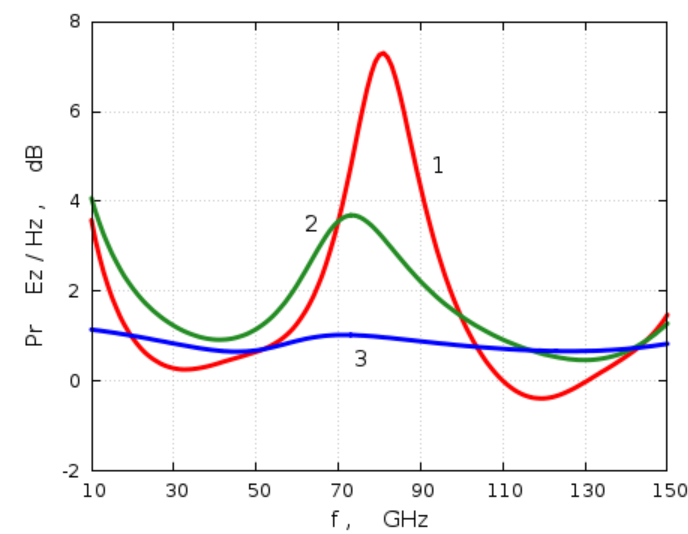

(b)

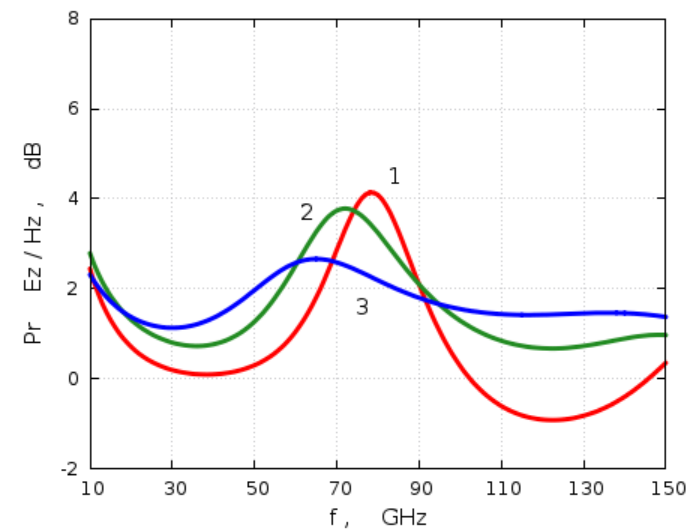

(c)

Figure 6: Polarization ratio for the waves reflected from (a) strip-like $\left(P_{L}=0.1,1,10 \mathrm{~kW} / \mathrm{cm}^{2}\right)$, (b) wire-like $\left(P_{L}=1,10,100 \mathrm{~kW} / \mathrm{cm}^{2}\right)$, and (c) fin-like $\left(P_{L}=\right.$ $0.1,0.3,1 \mathrm{~kW} / \mathrm{cm}^{2}$ ) photo-excited gratings (curves 1 to 3 , respectively).

at the very small diffusion length $L$, one can achieve a high contrast of photoconductivity, sharp transition from lossless intrinsic semiconductor domains to also lossless highconductivity wires, and minimal losses in the narrow shells of moderate conductivity around wires. At the low illumination, however, one still has to make sure that illumination is sufficient and the recombination length is small. This is 


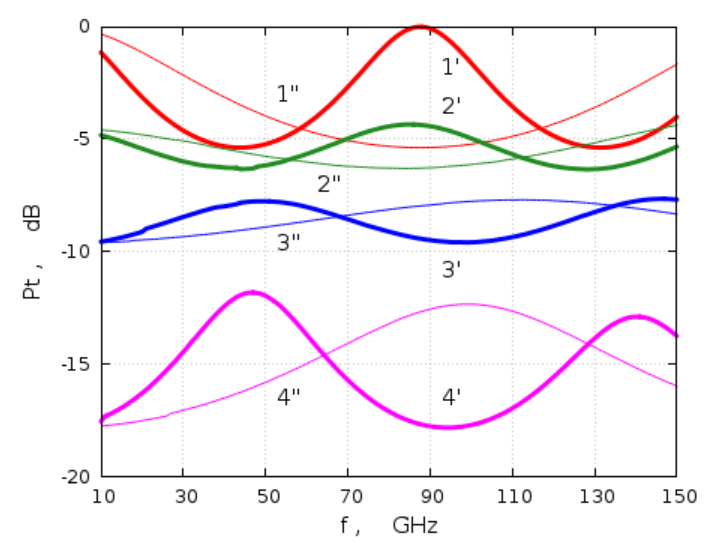

(a)

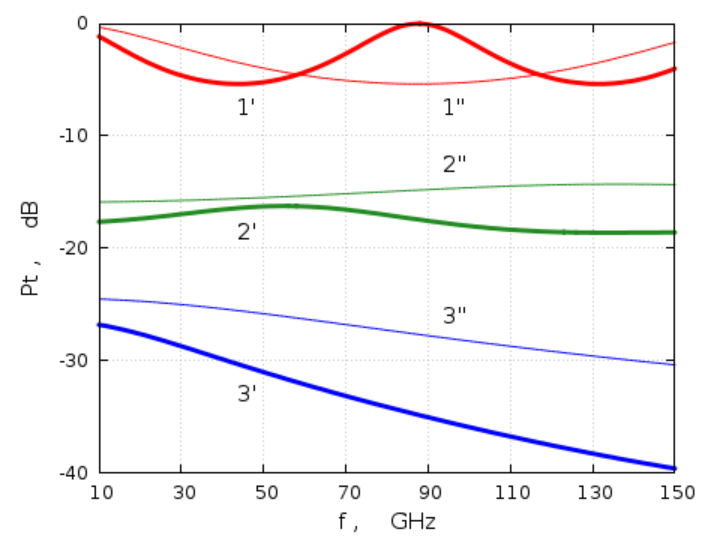

(b)

Figure 7: MM wave transmission through $0.5 \mathrm{~mm}$ and $0.25 \mathrm{~mm}$ Si wafer (prime and double-prime curve numbers, respectively) in the dark state (curves 1) and under the uniform (a) red light and (b) IR radiation with no grating mask at the light power fluxes $P_{L}=0.01,0.03,0.1 \mathrm{~kW} / \mathrm{cm}^{2}$ (curves 2 to 4 , respectively).

required for making the fin core domains sufficiently conductive (for the the wave scattering to be essential) and the fin shell domains reasonably thin (for absorption to remain minimal).

\section{Conclusions}

We investigated the functionality of photoconductive gratings in a semiconductor slab as light-controlled polarizers for MM waves. We performed numerical simulations of three characteristic kinds of such grating, which are the strip-like, the wire-like, and the fin-like gratings. The kind of gratings depends on the geometry of the opaque dielectric grid mask on the semiconductor surface, electron-hole diffusion length in a semiconductor, and the absorption length of the incident light exciting the gratings.

Simulations of photoconductive gratings in a semiconductor wafer of a resonant half-wavelength thickness transparent for MM waves have shown a possibility of making light-controlled MM wave polarizers at the moderate light intensity of, e.g., $P_{L}=0.1-10 \mathrm{~kW} / \mathrm{cm}^{2}$ that can be achieved with various light sources including, in particular, red light and IR radiation LED arrays.

At the same time, the simulations revealed some special effects such as the light-sensitive shift of maxima of transmitted power and polarizing efficiency of structures towards the lower frequency band as compared to wafers with no gratings in the dark state. The effect occurs due to the increase of refractive index in photoconductive domains so that the imaginary part $\varepsilon_{i}$ of the complex dielectric constant is getting greater than the real part $\varepsilon_{r}$. Then, the refractive index increase along with conductivity and, therefore, along with the light intensity.

The frequency shift is proportional to the degree of coupling between MM waves and gratings, which is greater for both the $E$ polarized waves and the fin-like gratings. Once the coupling has a complicated dependence on the grating profile and the light intensity, the shift would vary with the light intensity and may reverse under strong illumination, when MM waves do not penetrate anymore into conducting strips (this happens with increasing illumination in striplike and wire-like gratings, see curve 3 in Fig. 5, a).

The light-dependent spectral shift of the peak efficiency of photo-excited gratings makes them not so suitable for the application in the fixed-spectrum devices (except for the fin-like gratings at low illumination, whose spectrum is reasonably stable while the functionality is sufficient). On the contrary, the same effect makes photoconductive gratings and similar kinds of photo-excited conductivity patterns potentially suitable for the design of light-controlled frequency-tuning and frequency-modulating components of resonant quasi-optical devices.

\section{Acknowledgement}

The work was supported by The Scientific and Technological Research Council of Turkey (TUBITAK) through the 2221 Fellowship Program for Visiting Scientists and Scientists on Sabbatical Leave (2017/2).

\section{References}

[1] S. Busch, B. Scherger, M. Scheller, M. Koch, Optically controlled terahertz beam steering and imaging, Opt. Lett. 37: 8: 1391-1393, 2012.

[2] A. Kannegulla, Md. Itrat Bin Shams, L. Liu, L.-J. Cheng, Photo-induced spatial modulation of $\mathrm{THz}$ waves: opportunities and limitations, Opt. Express 23: 25: 32098-32112, 2015.

[3] L.-J. Cheng, L. Liu, Optical modulation of continuous terahertz waves towards cost-effective reconfigurable quasi-optical terahertz components, Opt. Express 21: 23: 28657-28667, 2013.

[4] X. Wang, Z. Xie, W. Sun, S. Feng, Y. Cui, J. Ye, Y. Zhang, Focusing and imaging of a virtual alloptical tunable terahertz Fresnel zone plate, Opt. Lett. 38: 22: 4731-4734, 2013. 
[5] Q. Zhou, Y. Shi, A. Wang, L. Li, D. Zhao, J. Liu, H. Sun, C. Zhang, Ultrafast optical modulation of terahertz metamaterials, J. Opt. 13: 11: 125102, 2011.

[6] Y. C. Jun, I. Brener, Electrically tunable infrared metamaterials based on depletion-type semiconductor devices, J. Opt. 14: 11: 114013, 2012.

[7] T. F. Gundogdu, M. Gökkavas, K. Güven, M. Kafesaki, C. M. Soukoulis, E. Ozbay, Simulation and micro-fabrication of optically switchable split ring resonators, Photonics Nanostruct. Fundam. Appl. 5: 106-112, 2007.

[8] G. Kenanakis, R. Zhao, N. Katsarakis, M. Kafesaki, C. M. Soukoulis, E. N. Economou, Optically controllable $\mathrm{THz}$ chiral metamaterials, Opt. Express 22: 10: 12149-12159, 2014.

[9] A. A. Vikharev, G. C. Denisov, V. V. Kocharovskii, S. V. Kuzikov, V. V. Parshin, N. Y. Peskov, A. N. Stepanov, D. I. Sobolev, M. Y. Shmelev, A highspeed quasi-optical wave phase switch based on the induced photoconductivity effect in Silicon, Tech. Phys. Lett. 33: 9: 735-737, 2007.

[10] I. F. D. Costa, D. H. Spadoti, A. C. Sodre Jr., A. L. D. Silva, J. S. R. Páez, R. P. Ramírez, V. Olmos, J. José, I. T. Monroy, Optically controlled reconfigurable antenna for $5 \mathrm{G}$ future broadband cellular communication networks, J. Microwaves, $\mathrm{Op}$ toelectronics and Electromagnetic Applications 16: 1: 208-217, 2017.

[11] J. Ren, Y. Deng, Y. Shi, A. Kannegulla, Y.-C. Wang, P. Fay, L.-J. Cheng, L. Liu, Optically controlled reconfigurable terahertz waveguide filters based on photo-induced electromagnetic band gap structures using mesa arrays, OSA Continuum 1: 4: 14291436, 2018.

[12] I. Chatzakis, P. Tassin, L. Luo, N. H. Shen, L. Zhang, J. Wang, T. Koschny, C. M. Soukoulis, Oneand two-dimensional photo-imprinted diffraction gratings for manipulating terahertz waves, Appl. Phys. Lett. 103: 043101, 2013.

[13] N. Yu, P. Genevet, M.A. Kats, F. Aieta, J.-P. Tetienne, F. Capasso, F. Gaburro, Light propagation with phase discontinuities: generalized laws of reflection and refraction, Science 334: 6054: 333$337,2011$.

[14] L. Fekete, F. Kadlec, P. Kuzel, H. Nemec, Ultrafast opto-terahertz photonic crystal modulator, Opt. Lett. 32: 6: 680-682, 2007.

[15] V. Yurchenko, M. Ciydem, M. Gradziel, A. Murphy, A. Altintas, Light-controlled photonics-based mm-wave beam switch, Opt. Express 24: 15: 16471, 2016.
[16] H. J. Eichler, Laser-induced Grating Phenomena, Optica Acta: Int. J. Opt. 24: 1977.

[17] A. Miller, D. A. B. Miller, S. D. Smith, Dynamic non-linear optical processes in semiconductors, Adv. Phys. 30: 6: 697-800, 1981.

[18] H. J. Eichler, P. Günter, D. W. Pohl, Laser-Induced Dynamic Gratings, Springer-Verlag, Berlin, 2013.

[19] H. J. Eichler, C. Hartig, J. Knof, Laser induced gratings in CdS, Phys. Stat. Sol. (a) 45: 2: 433-438, 1978.

[20] E. Gaubas, K. Jarašiũnas, J. Vaitkus, Light induced transient grating decay in $\mathrm{Si}$ and some AIIBIV compounds, Phys. Stat. Sol. (a) 69: 1: K87-K90, 1982.

[21] J. Vaitkus, K. Jarašiũnas, E. Gaubas, L. Jonikas, R. Paranaitis, L. Subačius, The diffraction of light by transient gratings in crystalline, ion-implanted, and amorphous Silicon, IEEE J. Quantum Electron. 22 8: 1298-1305, 1986.

[22] N. A. Kudryashov, S. S. Kucherenko, E. A. Mazur, A. N. Petrovskii, M. P. Yakovlev, Dynamics of light-induced diffraction gratings in Silicon excited by picosecond pulses, Sov. Phys. J. 33: 3: 246-250, 1990.

[23] M. A. Bryushinin, P. M. Karavaev, I. A. Sokolov, Optically induced space-charge and conductivity gratings in wide-bandgap semiconductors, Phys. Procedia 86: 136-140, 2017.

[24] M.A. Green, M.J. Keevers, Optical properties of intrinsic Silicon at $300 \mathrm{~K}$, Prog. Photovoltaics Res. Appl. 3: 3: 189-192, 1995.

[25] S.M. Sze, Semiconductor Devices, Physics and Technology, John Wiley and Sons, Hoboken, NJ, 2002.

[26] J.-M. Jin, The Finite Element Method in Electromagnetics, 3rd Edition, John Wiley and Sons, Hoboken, NJ, 2015.

[27] V.B. Yurchenko, M.L. Gradziel, J.A. Murphy, Dual-layer grid polarizers for $\mathrm{mm}$ and sub-mm waves: theory and experiment, Proc. MSMW'2010, Kharkov, Ukraine, W-5, 2010. 